\title{
Safety and efficacy of durvalumab (MEDI4736) in various solid tumors
}

This article was published in the following Dove Press journal:

Drug Design, Development and Therapy

\author{
Hui Yang ${ }^{1,2, *}$ \\ Kai Shen ${ }^{3, *}$ \\ Chenjing Zhu ${ }^{3}$ \\ Qingfang $\mathrm{Li}^{3}$ \\ Yunuo Zhao ${ }^{2}$ \\ Xuelei $\mathrm{Ma}^{3, *}$
}

'Cancer Center, West China Hospital, Sichuan University, Chengdu, People's Republic of China; ${ }^{2}$ West China School of Medicine, West China Hospital, Sichuan University, Chengdu, People's Republic of China; ${ }^{3}$ State Key Laboratory of Biotherapy and Cancer Center, West China Hospital, Sichuan University and Collaborative Innovation Center, Chengdu, People's Republic of China

*These authors contributed equally to this work
Correspondence: Xuelei Ma State Key Laboratory of Biotherapy and Cancer Center, West China Hospital, Sichuan University and Collaborative Innovation Center, 37 Guoxue Alley, Chengdu 61004I, People's Republic of China Tel/fax +862885475576 Email drmaxuelei@gmail.com
Introduction: The prominent immune checkpoint molecule, programmed cell death ligand-1 (PD-L1), is the object of increasing attention. Here, we report a meta-analysis investigating the safety and efficacy of durvalumab (MEDI4736), an inhibitor of PD-L1, in various solid tumors.

Methods: A systematic search of PubMed, Embase, and related articles was performed. Safety data were analyzed using Comprehensive Meta-Analysis software program version 2. Ultimately, 17 studies with 1,529 patients were included in our analysis.

Results: The major adverse events associated with durvalumab were pruritus and fatigue, while pruritus, increased alanine transaminase, and increased aspartate aminotransferase were common among patients treated with a combination of durvalumab and tremelimumab. Higher PD-L1 expression was associated with a superior objective response rate.

Conclusion: Durvalumab is safe in patients with many solid cancers and, in combination with tremelimumab, it has a tolerable safety profile and is associated with improved prognosis. PD-L1 expression is a biomarker of the efficacy of durvalumab.

Keywords: durvalumab, solid cancers, adverse effects, efficacy, meta-analysis

\section{Introduction}

The American Cancer Society recently published data predicting that 1,688,780 new cancer cases and 600,920 cancer-related deaths would occur in the USA in $2017 .{ }^{1}$ Newly developing therapies for cancer are increasing and serve to complement traditional approaches, such as surgery, chemotherapy, and radiotherapy. Among emerging therapies, immunotherapy is particularly noteworthy, and there are several ongoing trials for this approach. ${ }^{2}$

An important feature of cancer cells is their ability to escape from immune surveillance by interacting with T-cell receptors. ${ }^{3}$ Such interactions can hinder T-cell immunity and help cancer cells escape from protective immune responses, referred to as immune checkpoints. ${ }^{4}$ Two vital immune checkpoint-associated molecules are programmed cell death ligand-1 (PD-L1 or CD274) and cytotoxic T-lymphocyte-associated antigen-4 (CTLA-4 or CD152). Programmed cell death-1 (PD-1 or CD279) is a receptor present on activated T cells, while PD-L1 is expressed, or overexpressed, on the surfaces of various cancer cells. ${ }^{4,5}$ On formation, the PD-L1 and PD-1 complex releases signals that have inhibitory effects on T cells. These inhibitory signals can suppress T-cellmediated immunity and may lead to tumor progression., ${ }^{5,6}$ CTLA-4 interacts with B7, which is expressed on antigen-presenting cells, ${ }^{4}$ that also physically interacts with the costimulatory factor, CD28. Hence, the interaction of CTLA-4 and B7 can impede T-cell activation by blocking the contact between CD28 and B7. ${ }^{7}$ 
The blockade of immune checkpoints can facilitate the recognition of cancer cells by an organism, allowing it to enhance antitumor immunity accordingly. Inhibitors function in both the priming phase (CTLA-4/B7) and the effector phase (PD-1/PD-L1) of immune cell (IC) cycles. ${ }^{4}$ CTLA-4 inhibitors were approved for clinical use in 2011, the year when ipilimumab was first used for the treatment of unresectable or metastatic melanoma. ${ }^{8}$ Durvalumab, a human

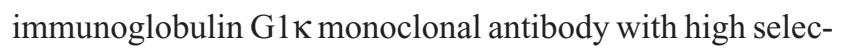
tivity and affinity, can block the binding of PD-L1 to PD-1 and CD80. ${ }^{9}$ The US Food and Drug Administration (FDA) granted breakthrough therapy designation to durvalumab in February 2016 for patients with inoperable or metastatic PDL1-positive urothelial bladder cancer and cancer progression following chemotherapy. ${ }^{3}$ As a PD-L1 inhibitor, durvalumab can be used alone or in combination with other therapies, such as chemotherapy, radiotherapy, targeted therapy, or other immunotherapy. We conducted this meta-analysis to evaluate the safety and efficacy of durvalumab for the treatment of various cancers.

\section{Materials and methods}

\section{Literature search}

Articles were identified by searching PubMed and Embase using the keywords "MEDI4736", "durvalumab", or "Imfinzi" (publications from 1974 to September 24, 2017). Relevant articles were also obtained by searching the reference list of primary articles or via relevant clinical trial information in American Society of Clinical Oncology (ASCO) and European Society for Medical Oncology (ESMO) Congress databases.

\section{Inclusion and exclusion criteria}

Studies eligible for inclusion met the following criteria: 1) clinical trials in any phase concerning durvalumab, durvalumab plus tremelimumab, or durvalumab plus other targeted drugs; 2 ) patients involved had pathologically confirmed cancer; and 3) adverse events (AEs) or efficacy data were reported.

Studies were excluded if they met one of the following conditions: 1) no raw data; 2 ) lack of adequate data to evaluate the efficacy or safety of durvalumab; and 3) reviews, editorials, cases, letters, errata, or nonhuman studies.

To avoid duplication of data, we chose articles with more useful data rather than the most recent publications or those including more patients.

\section{Data extraction}

Two authors (HY and KS) independently considered eligible articles and extracted data. Disagreements were resolved by discussing with a third reviewer. The following data were extracted from eligible articles: 1) the basic characteristics of studies, including first author, year of publication, clinical trial information, study phase, treatment, number of participants, and type of cancer; 2) AEs that appeared in at least two papers; and 3) efficacy data including median progression-free survival (mPFS), median overall survival (mOS), complete response, partial response (PR), stable disease, and objective response rate (ORR).

\section{Statistical analysis}

Safety data analysis was performed using the Comprehensive Meta-Analysis software program version 2 (CMA V2, Biostat, Englewood, NJ, USA). We calculated the percentage and derived $95 \%$ confidence interval (CI) for any grade AEs in each study. A random-effects model was applied, where $I^{2} \geq 50 \%$.

\section{Results \\ Eligible articles}

A total of 109 articles and conference reports were assessed, of which 17 were eligible. ${ }^{10-26}$ Three articles had the same clinical trial number (NCT01693562); however, each focused on a different tumor type. ${ }^{11,14,15}$ Clinical trial NCT02141347 was designed to investigate the safety of combining durvalumab with tremelimumab; however, only the results for durvalumab were provided. ${ }^{19}$ Eight articles on durvalumab and nine articles on durvalumab along with another drug were eligible for the final analysis; the detailed process of study selection is presented in Figure 1. Eligible articles included two Phase II, nine Phase I, and five Phase I/II studies, and they were all published between 2015 and 2017. The basic data from the included studies are shown in Table 1.

In total, 17 studies with 1,529 patients were included in our analysis. The major AEs associated with durvalumab were pruritus and fatigue. Pruritus, increased alanine transaminase (ALT), and increased aspartate aminotransferase (AST) were commonly recorded for patients treated with a combination of durvalumab and tremelimumab. According to our analysis, higher PD-L1 expression was associated with superior ORR.

\section{Safety analysis}

In our analysis, the major AEs associated with durvalumab were pruritus, fatigue, decreased appetite, diarrhea, increased AST, nausea, and rash. A random-effects model was used for analyses of increased ALT, increased AST, leukopenia, and pruritus. Pruritus had the highest overall event rate 


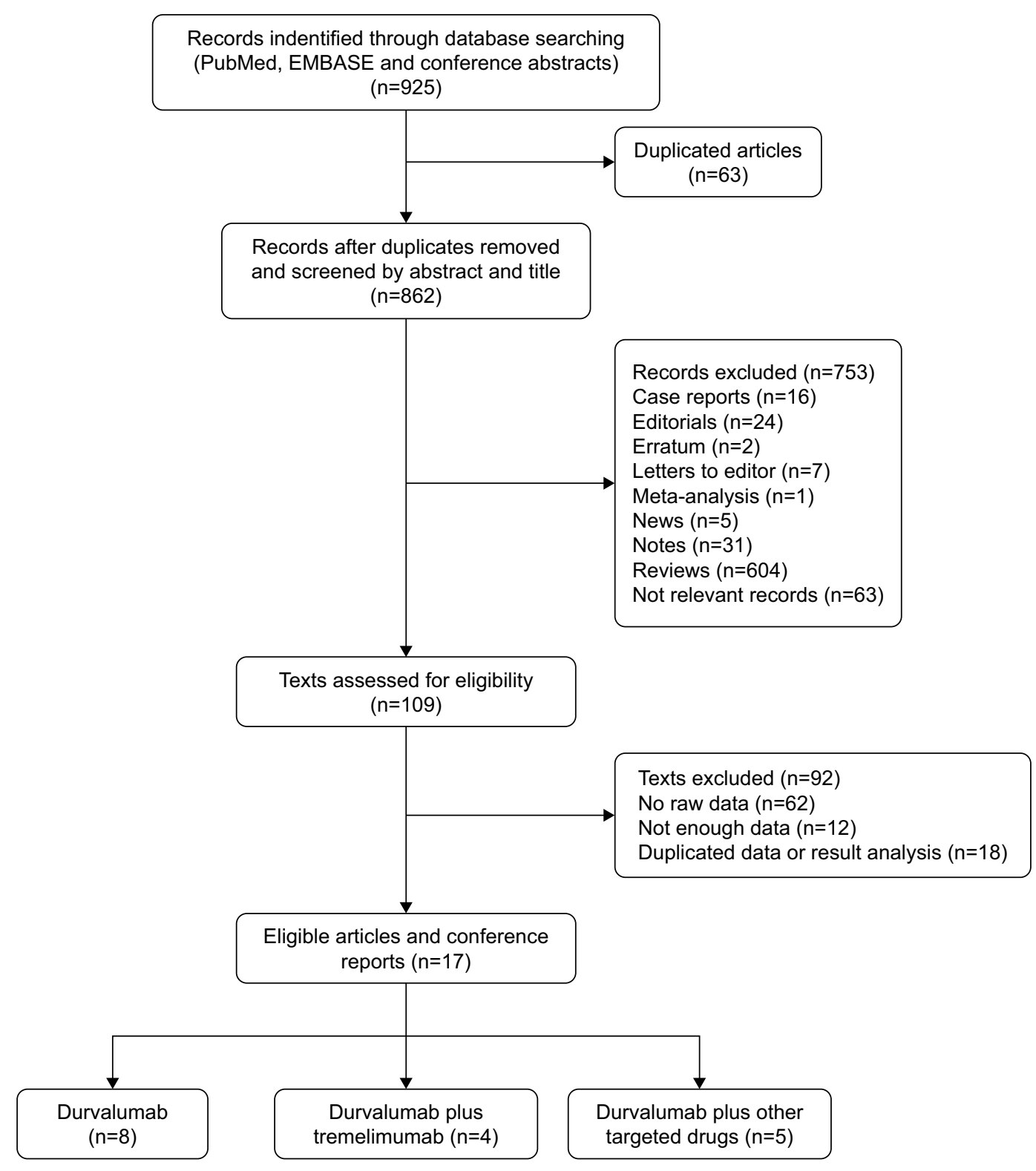

Figure I Flow chart illustrating the article searching process used for this study.

of 0.146 (95\% CI: 0.041-0.405), followed by fatigue (0.190 [95\% CI: 0.161-0.224]), and decreased appetite (0.097 [95\% CI: 0.075-0.125]). Meanwhile, diarrhea and increased AST occurred at similar rates (0.090 vs 0.089$)$. Common grade $>3$ AEs for durvalumab alone were decreased appetite (0.055 [95\% CI: 0.013-0.196]) and increased AST (0.034 [95\% CI: 0.007-0.149]). In trials combining durvalumab with tremelimumab, the most common AEs were pruritus (0.202 [95\% CI: 0.143-0.277]), increased ALT (0.126 [95\% CI: 0.080-0.193]), and increased AST (0.105 [95\% CI: 0.63-0.169]) (Figure 2). Serious AEs that led to the discontinuation of the study drug were reported in 10 papers (Table 2). ${ }^{10,14,16,17,19-22,24,25}$ TATTON (NCT02143466) reported that grade 3-4 interstitial lung disease (ILD) occurred in
$14.7 \%(5 / 34)$ of patients with epidermal growth factor receptor $(E G F R)$-positive advanced non-small-cell lung cancer (NSCLC) treated with a combination of durvalumab and AZD9291; this combination arm was terminated due to an increased incidence of ILD. ${ }^{20}$

\section{Efficacy analysis}

There were five studies related to the efficacy of durvalumab. ${ }^{11,12,15,16,25}$ Study NCT02087423 measured the expression of PD-L1 on tumor cell (TC) membranes in NSCLC patients and chose $25 \%$ as the cutoff value. The PD-L1-positive subgroup had higher ORR (16.4\% vs 7.5\%), mPFS (3.3 vs 1.9 months), and mOS (10.9 vs 9.3 months) values than the PD-L1-negative subgroup. ${ }^{16}$ When $90 \%$ was 
Table I Basic characteristics of the included studies

\begin{tabular}{|c|c|c|c|c|c|}
\hline $\begin{array}{l}\text { Author, } \\
\text { year }\end{array}$ & $\begin{array}{l}\text { Clinical trial } \\
\text { information }\end{array}$ & Study title & Phase & $\begin{array}{l}\text { Participants } \\
\text { (N) }\end{array}$ & Disease \\
\hline $\begin{array}{l}\text { Iguchi et al, } \\
2015^{25}\end{array}$ & NCT0I9386I2 & $\begin{array}{l}\text { A Phase I, open-label, multicenter study to evaluate the safety, tolerability, } \\
\text { and pharmacokinetics of MEDI } 4736 \text { in patients with advanced solid tumors. }\end{array}$ & 1 & 22 & $\begin{array}{l}\text { Advanced solid } \\
\text { tumors }\end{array}$ \\
\hline $\begin{array}{l}\text { Ribas et al, } \\
2015^{24}\end{array}$ & NCT0202796I & $\begin{array}{l}\text { A Phase I open-label study on safety and tolerability of MEDI } 4736 \text { in } \\
\text { subjects with metastatic or unresectable melanoma in combination with } \\
\text { dabrafenib and trametinib or with trametinib alone. }\end{array}$ & I & 50 & $\begin{array}{l}\text { Advanced } \\
\text { melanoma }\end{array}$ \\
\hline $\begin{array}{l}\text { Takahashi } \\
\text { et al, } 2015^{19}\end{array}$ & NCT02। 4 I 347 & $\begin{array}{l}\text { A Phase I open-label multicenter study to assess safety, tolerability, } \\
\text { pharmacokinetics, and antitumor activity of tremelimumab/tremelimumab } \\
\text { with MEDI } 4736 \text { in Japanese with advanced solid malignancies or } \\
\text { tremelimumab in Japanese with malignant mesothelioma. }\end{array}$ & I & 8 & $\begin{array}{l}\text { Advanced solid } \\
\text { malignancies }\end{array}$ \\
\hline $\begin{array}{l}\text { Lee et al, } \\
2017^{23}\end{array}$ & NCT02484404 & $\begin{array}{l}\text { Phase } 1 / I \text { study of the antiprogrammed death ligand-I antibody MEDI } 4736 \\
\text { in combination with olaparib and/or cediranib for advanced solid tumors } \\
\text { and advanced or recurrent ovarian, triple-negative breast, lung, prostate, } \\
\text { and colorectal cancers. }\end{array}$ & I & 26 & $\begin{array}{l}\text { Recurrent } \\
\text { women's } \\
\text { cancers }\end{array}$ \\
\hline $\begin{array}{l}\text { Garassino } \\
\text { et al, } 2017^{16}\end{array}$ & NCT02087423 & $\begin{array}{l}\text { A Phase II, noncomparative, open-label, multicenter, international study of } \\
\text { MEDI4736 in patients with locally advanced or metastatic non-small cell } \\
\text { lung cancer (stage IIIB-IV) who have received at least two prior systemic } \\
\text { treatment regimens, including one platinum-based chemotherapy regimen. }\end{array}$ & II & 333 & $\begin{array}{l}\text { Advanced or } \\
\text { metastatic } \\
\text { stage IIIB-IV } \\
\text { NSCLC }\end{array}$ \\
\hline $\begin{array}{l}\text { Ahn et al, } \\
2017^{20}\end{array}$ & NCT02। 43466 & $\begin{array}{l}\text { A multiarm, Phase lb, open-label, multicenter study to assess the safety, } \\
\text { tolerability, pharmacokinetics, and preliminary antitumor activity of } \\
\text { AZD929I in combination with ascending doses of novel therapeutics } \\
\text { in patients with EGFR-mutant advanced NSCLC who have progressed } \\
\text { following therapy with an EGFR TKI (TATTON). }\end{array}$ & $\mathrm{lb}$ & 34 & $\begin{array}{l}\text { EGFR-mutant } \\
\text { NSCLC }\end{array}$ \\
\hline $\begin{array}{l}\text { Gibbons } \\
\text { et al, } 2016^{22}\end{array}$ & NCT02088II 2 & $\begin{array}{l}\text { A Phase I, open-label, multicenter study to assess the safety, tolerability, } \\
\text { pharmacokinetics, and preliminary antitumor activity of gefitinib in } \\
\text { combination with MEDI } 4736 \text { (anti-PD-LI) in subjects with NSCLC. }\end{array}$ & I & 20 & $\begin{array}{l}\text { TKI-naive with } \\
\text { EGFR mutant } \\
\text { NSCLC }\end{array}$ \\
\hline $\begin{array}{l}\text { Powles } \\
\text { et al, } 2017^{17}\end{array}$ & NCT0I693562 & $\begin{array}{l}\text { A Phase I/II study to evaluate the safety, tolerability, and pharmacokinetics } \\
\text { of MEDI4736 in subjects with advanced solid tumors. }\end{array}$ & $\mathrm{I} / \mathrm{II}$ & 191 & $\begin{array}{l}\text { Locally } \\
\text { advanced or } \\
\text { metastatic } \\
\text { UCC }\end{array}$ \\
\hline $\begin{array}{l}\text { Santa-Maria } \\
\text { et al, } 2017^{26}\end{array}$ & NCT02536794 & $\begin{array}{l}\text { A single-arm Phase II study evaluating the efficacy and safety of } \\
\text { MEDI4736 in combination with tremelimumab in patients with metastatic } \\
\text { Her2-negative breast cancer. }\end{array}$ & NM & 18 & $\begin{array}{l}\text { Metastatic } \\
\text { breast cancer }\end{array}$ \\
\hline $\begin{array}{l}\text { Kelley et al, } \\
2017^{10}\end{array}$ & NCT025I9348 & $\begin{array}{l}\text { A study of safety, tolerability, and clinical activity of MEDI4736 and } \\
\text { tremelimumab administered as monotherapy and in combination to } \\
\text { subjects with unresectable hepatocellular carcinoma. }\end{array}$ & $\mathrm{I} / \mathrm{II}$ & 40 & $\begin{array}{l}\text { Unresectable } \\
\text { HCC }\end{array}$ \\
\hline $\begin{array}{l}\text { Wainberg } \\
\text { et al, 2017"1 }\end{array}$ & NCT0I693562 & $\begin{array}{l}\text { A Phase I/II study to evaluate the safety, tolerability, and pharmacokinetics } \\
\text { of MEDI } 4736 \text { in subjects with advanced solid tumors. }\end{array}$ & $\mathrm{I} / \mathrm{II}$ & 40 & $\begin{array}{l}\text { Advanced } \\
\text { HCC }\end{array}$ \\
\hline $\begin{array}{l}\text { Callahan } \\
\text { et al, } 2017^{18}\end{array}$ & NCTOI97583I & $\begin{array}{l}\text { A Phase I study to evaluate the safety and tolerability of anti-PD-LI, } \\
\text { MEDI } 4736 \text {, in combination with tremelimumab in subjects with advanced } \\
\text { solid tumors. }\end{array}$ & I & 105 & $\begin{array}{l}\text { Advanced solid } \\
\text { tumors }\end{array}$ \\
\hline $\begin{array}{l}\text { Reardon } \\
\text { et al, } 2017^{12}\end{array}$ & NCT02336I65 & $\begin{array}{l}\text { Phase II study to evaluate the clinical efficacy and safety of MEDI } 4736 \text { in } \\
\text { patients with glioblastoma. }\end{array}$ & II & 154 & Glioblastoma \\
\hline $\begin{array}{l}\text { Lin et al, } \\
2016^{13}\end{array}$ & NCT02572687 & $\begin{array}{l}\text { An open-label, multicenter, Phase I study of ramucirumab plus MEDI } 4736 \\
\text { in patients with locally advanced and unresectable or metastatic } \\
\text { gastrointestinal or thoracic malignancies. }\end{array}$ & la & 20 & $\begin{array}{l}\text { Advanced } \\
\text { gastrointestinal } \\
\text { or thoracic } \\
\text { malignancies }\end{array}$ \\
\hline $\begin{array}{l}\text { Antonia } \\
\text { et al, } 2016^{14}\end{array}$ & NCT0I693562 & $\begin{array}{l}\text { A Phase I/II study to evaluate the safety, tolerability, and pharmacokinetics } \\
\text { of MEDI } 4736 \text { in subjects with advanced solid tumors. }\end{array}$ & $\mathrm{I} / \mathrm{II}$ & 304 & NSCLC \\
\hline $\begin{array}{l}\text { Antonia } \\
\text { et al, } 2016^{21}\end{array}$ & NCT02000947 & $\begin{array}{l}\text { A Phase lb open-label study to evaluate the safety and tolerability } \\
\text { of MEDI } 4736 \text { in combination with tremelimumab in subjects with } \\
\text { advanced NSCLC. }\end{array}$ & $\mathrm{lb}$ & 102 & $\begin{array}{l}\text { Advanced } \\
\text { NSCLC }\end{array}$ \\
\hline $\begin{array}{l}\text { Segal et al, } \\
2016^{15}\end{array}$ & NCT0I693562 & $\begin{array}{l}\text { A Phase I/II study to evaluate the safety, tolerability, and pharmacokinetics } \\
\text { of MEDI4736 in subjects with advanced solid tumors. }\end{array}$ & $\mathrm{I} / \mathrm{II}$ & 62 & $\begin{array}{l}\text { Recurrent and } \\
\text { metastatic } \\
\text { SCCHN }\end{array}$ \\
\hline
\end{tabular}

Abbreviations: EGFR, epidermal growth factor receptor; HCC, hepatocellular carcinoma; Her2, human epidermal growth factor receptor 2; NM, not mentioned; NSCLC, non-small-cell lung cancer; PD-LI, programmed cell death ligand-I; SCCHN, squamous cell carcinoma of head and neck; TKI, tyrosine kinase inhibitor; UCC, urothelial carcinoma. 
used as the cutoff value, ORR implausibly reached $30.9 \%$ (95\% CI: 20.2-43.3) in the positive group, while mPFS was only 2.4 months (95\% CI: $1.8-5.5) .{ }^{20}$ The other four studies, including 207 patients treated with durvalumab and tremelimumab, reported different ORRs ranging from $15 \%$ to $38 \%$ (Tables 3 and 4 ). ${ }^{10,18,21,26}$

\section{Discussion}

Intravenous durvalumab (Imfinzi ${ }^{\mathrm{TM}}$; AstraZeneca, Cambridge, UK) received US FDA-accelerated approval for previously treated advanced bladder cancer in May 2017. ${ }^{27}$ It was reported to dramatically extend the mPFS of patients with stage III NSCLC after concurrent chemoradiotherapy
A

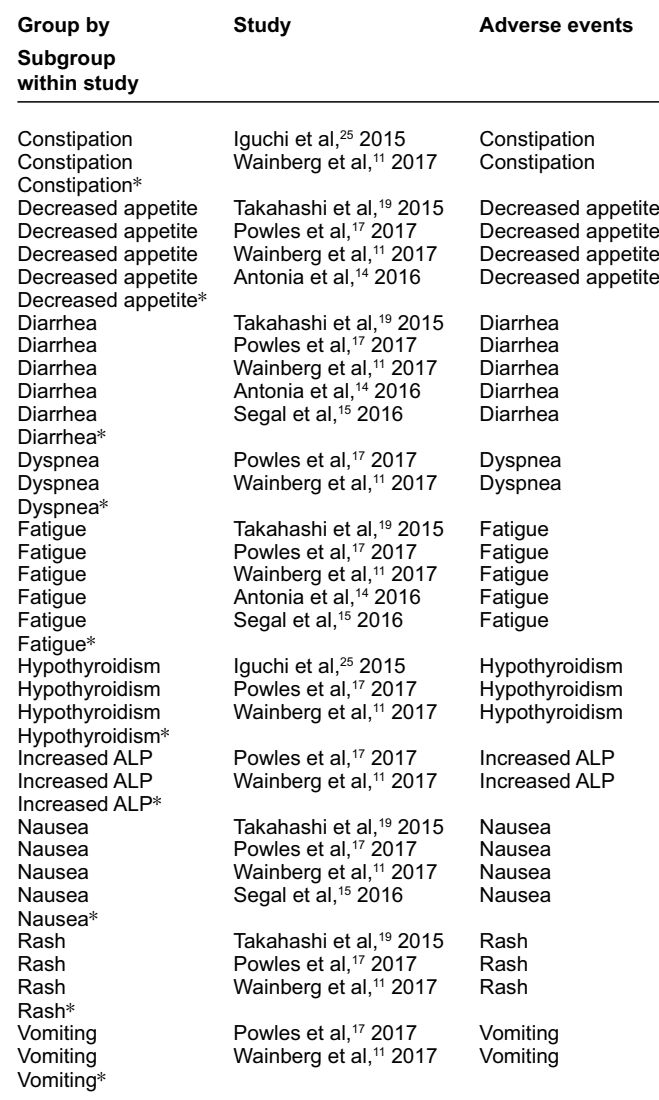

Meta analysis

Statistics for each study

Event Lower Upper

rate limit limit Z-value $P$-value

$\begin{array}{lllll}0.045 & 0.006 & 0.261 & -2.975 & 0.003\end{array}$

$\begin{array}{lllll}0.045 & 0.006 & 0.261 & -2.975 & 0.003 \\ 0.075 & 0.024 & 0.208 & -4.185 & 0.000\end{array}$

$\begin{array}{lllll}0.075 & 0.024 & 0.208 & -4.185 & 0.000 \\ 0.066 & 0.025 & 0.163 & -5.115 & 0.000\end{array}$

$\begin{array}{lllll}0.066 & 0.025 & 0.163 & -5.115 & 0.000 \\ 0.250 & 0.063 & 0.623 & -1.346 & 0.178\end{array}$

$\begin{array}{lllll}0.250 & 0.063 & 0.623 & -1.346 & 0.178 \\ 0.094 & 0.060 & 0.145 & -9.137 & 0.000\end{array}$

$\begin{array}{lllll}0.094 & 0.060 & 0.145 & -9.137 & 0.000 \\ 0.125 & 0.053 & 0.267 & -4.070 & 0.000\end{array}$

$\begin{array}{lllll}0.125 & 0.053 & 0.267 & -4.070 & 0.000 \\ 0.089 & 0.062 & 0.126 & -11.548 & 0.000\end{array}$

$\begin{array}{lllll}0.089 & 0.062 & 0.126 & -11.548 & 0.000 \\ 0.097 & 0.075 & 0.125 & -15.254 & 0.000\end{array}$

$\begin{array}{lllll}0.097 & 0.075 & 0.125 & -15.254 & 0.000 \\ 0.250 & 0.063 & 0.623 & -1.346 & 0.178\end{array}$

$\begin{array}{lllll}0.250 & 0.063 & 0.623 & -1.346 & 0.178 \\ 0.084 & 0.052 & 0.132 & -9.159 & 0.000\end{array}$

$\begin{array}{lllll}0.084 & 0.052 & 0.132 & -9.159 & 0.000 \\ 0.100 & 0.038 & 0.238 & -4.169 & 0.000\end{array}$

$\begin{array}{lllll}0.100 & 0.038 & 0.238 & -4.169 & 0.000 \\ 0.089 & 0.062 & 0.126 & -11.548 & 0.000\end{array}$

$\begin{array}{lllll}0.089 & 0.062 & 0.126 & -11.548 & 0.000 \\ 0.081 & 0.034 & 0.180 & -5.218 & 0.000\end{array}$

$\begin{array}{lllll}0.090 & 0.070 & 0.116 & -16.163 & 0.000\end{array}$

$\begin{array}{lllll}0.021 & 0.008 & 0.116 & -16.163 & 0.000 \\ 0.021 & 0.054 & -7.609 & 0.000\end{array}$

$\begin{array}{lllll}0.050 & 0.013 & 0.179 & -7.609 & 0.000 \\ 0.028 & 0.013 & 0.061 & -8.563 & 0.000\end{array}$

$\begin{array}{lllll}0.028 & 0.013 & 0.061 & -8.563 & 0.000\end{array}$

$\begin{array}{lllll}0.375 & 0.125 & 0.715 & -0.563 & 0.000 \\ 0.194 & 0.144 & 0.256 & -7.789 & 0.484\end{array}$

$\begin{array}{lllll}0.194 & 0.144 & 0.256 & -7.789 & 0.000\end{array}$

$\begin{array}{lllll}0.275 & 0.159 & 0.432 & -2.738 & 0.006\end{array}$

$\begin{array}{lllll}0.171 & 0.133 & 0.218 & -10.362 & 0.000\end{array}$

$\begin{array}{lllll}0.177 & 0.133 & 0.218 & -10.362 & 0.000 \\ 0.101 & 0.293 & -4.614 & 0.000\end{array}$

$\begin{array}{lllll}0.177 & 0.101 & 0.293 & -4.614 & 0.000 \\ 0.190 & 0.161 & 0.224 & -13.893 & 0.000\end{array}$

$\begin{array}{lllll}0.190 & 0.161 & 0.224 & -13.893 & 0.000 \\ 0.045 & 0.006 & 0.261 & -2.975 & 0.003\end{array}$

$\begin{array}{lllll}0.045 & 0.006 & 0.261 & -2.975 & 0.003 \\ 0.047 & 0.025 & 0.088 & -8.805 & 0.000\end{array}$

$\begin{array}{lllll}0.047 & 0.025 & 0.088 & -8.805 & 0.000 \\ 0.050 & 0.013 & 0.179 & -4.059 & 0.000 \\ 0.047 & 0.027 & 0.082 & -10.141 & 0.000\end{array}$

$\begin{array}{lllll}0.050 & 0.013 & 0.179 & -4.059 & 0.000 \\ 0.047 & 0.027 & 0.082 & -10.141 & 0.000\end{array}$

$\begin{array}{lllll}0.047 & 0.027 & 0.082 & -10.141 & 0.000 \\ 0.021 & 0.008 & 0.054 & -7.609 & 0.000\end{array}$

$\begin{array}{lllll}0.021 & 0.008 & 0.054 & -7.609 & 0.000 \\ 0.050 & 0.013 & 0.179 & -4.059 & 0.000\end{array}$

$\begin{array}{lllll}0.050 & 0.013 & 0.179 & -4.059 & 0.000 \\ 0.028 & 0.013 & 0.061 & -8.563 & 0.000\end{array}$

$\begin{array}{lllll}0.250 & 0.063 & 0.061 & -8.563 & 0.000 \\ 0.068 & 0.040 & 0.114 & -1.346 & 0.178\end{array}$

$\begin{array}{lllll}0.068 & 0.040 & 0.114 & -9.108 & 0.000\end{array}$

$\begin{array}{lllll}0.100 & 0.038 & 0.238 & -4.169 & 0.000\end{array}$

$\begin{array}{lllll}0.081 & 0.034 & 0.180 & -5.218 & 0.000\end{array}$

$\begin{array}{lllll}0.083 & 0.056 & 0.121 & -11.230 & 0.000\end{array}$

$\begin{array}{lllll}0.250 & 0.063 & 0.623 & -1.346 & 0.178\end{array}$

$\begin{array}{lllll}0.073 & 0.044 & 0.120 & -9.138 & 0.000\end{array}$

$\begin{array}{lllll}0.075 & 0.024 & 0.208 & -4.185 & 0.000\end{array}$

$\begin{array}{lllll}0.083 & 0.053 & 0.126 & -10.001 & 0.000\end{array}$

$\begin{array}{lllll}0.031 & 0.014 & 0.068 & -8.265 & 0.000 \\ 0.075 & 0.024 & 0.208 & -4.185 & 0.000\end{array}$

$\begin{array}{lllll}0.075 & 0.024 & 0.208 & -4.185 & 0.000 \\ 0.042 & 0.022 & 0.078 & -9.179 & 0.000\end{array}$
Event rate and $95 \% \mathrm{Cl}$

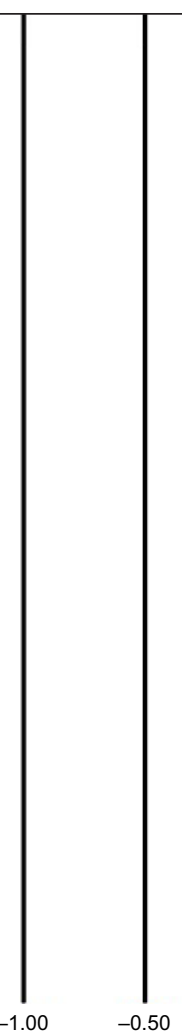

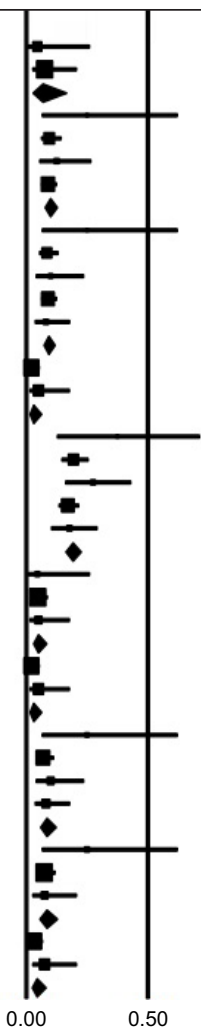

\section{B}

\begin{tabular}{|c|c|c|}
\hline $\begin{array}{l}\text { Group by } \\
\text { Subgroup } \\
\text { within study }\end{array}$ & Study & Adverse events \\
\hline Increased ALT & Powles et al, ${ }^{17} 2017$ & Increased ALT \\
\hline Increased ALT & Wainberg et al, ${ }^{11} 2017$ & Increased ALT \\
\hline \multicolumn{3}{|l|}{ Increased ALT* } \\
\hline Increased AST & Powles et al, ${ }^{17} 2017$ & Increased AST \\
\hline Increased AST & Wainberg et al, ${ }^{11} 2017$ & Increased AST \\
\hline \multicolumn{3}{|l|}{ Increased AST* } \\
\hline Leukopenia & Powles et al, ${ }^{17} 2017$ & Leukopenia \\
\hline Leukopenia & Wainberg et al, ${ }^{11} 2017$ & Leukopenia \\
\hline \multicolumn{3}{|l|}{ Leukopenia* } \\
\hline Pruritus & Takahashi et al, ${ }^{19} 2015$ & Pruritus \\
\hline Pruritus & Powles et al, ${ }^{17} 2017$ & Pruritus \\
\hline Pruritus & Wainberg et al, ${ }^{11} 2017$ & Pruritus \\
\hline Pruritus* & & \\
\hline
\end{tabular}

Meta analysis

Statistics for each study

Event Lower Upper

rate limit limit Z-value $P$-value

$\begin{array}{lllll}0.042 & 0.021 & 0.082 & -8.666 & 0.000\end{array}$

$\begin{array}{lllll}0.100 & 0.038 & 0.238 & -4.169 & 0.000\end{array}$

$\begin{array}{lllll}0.061 & 0.025 & 0.137 & -5.966 & 0.000\end{array}$

$\begin{array}{lllll}0.031 & 0.014 & 0.068 & -8.265 & 0.000\end{array}$

$\begin{array}{lllll}0.225 & 0.121 & 0.379 & -3.266 & 0.001\end{array}$

$\begin{array}{lllll}0.089 & 0.011 & 0.456 & -2.123 & 0.034\end{array}$

$\begin{array}{lllll}0.005 & 0.001 & 0.036 & -5.233 & 0.000\end{array}$

$\begin{array}{lllll}0.050 & 0.013 & 0.179 & -4.059 & 0.000\end{array}$

$\begin{array}{lllll}0.018 & 0.002 & 0.149 & -3.481 & 0.000\end{array}$

$\begin{array}{lllll}0.250 & 0.063 & 0.623 & -1.346 & 0.178\end{array}$

$\begin{array}{lllll}0.052 & 0.028 & 0.095 & -8.915 & 0.000\end{array}$

$\begin{array}{lllll}0.250 & 0.140 & 0.405 & -3.009 & 0.003\end{array}$

$\begin{array}{llll}0.146 & 0.041 & 0.405 & -2.502\end{array}$

0.012

Figure 2 (Continued) 
C

\begin{tabular}{l} 
Group by \\
Subgroup \\
within study \\
\hline Decreased appetite \\
Decreased appetite \\
Decreased appetite* \\
Fatigue \\
Fatigue \\
Fatigue* \\
Increased ALP \\
Increased ALP \\
Increased ALP*
\end{tabular}

Study

Takahashi et al 192015

Takahashi et al, ${ }^{19} 2015$ Wainberg et al, ${ }^{11} 2017$

Wainberg et al, ${ }^{11} 2017$ Antonia et al, ${ }^{14} 2016$

Powles et al, ${ }^{17} 2017$ Wainberg et al, ${ }^{11} 2017$
Adverse events grade $>3$

(Drade $>3$

Decreased appetite Decreased appetite

Fatigue

Fatigue

Increased ALP Increased ALP
Meta analysis

Statistics for each study Event Lower Upper rate limit limit

$\begin{array}{lll}0.125 & 0.017 & 0.537\end{array}$
$\begin{array}{lll}0.125 & 0.017 & 0.537 \\ 0.025 & 0.004 & 0.157\end{array}$ $\begin{array}{lll}0.025 & 0.004 & 0.157 \\ 0.055 & 0.013 & 0.196\end{array}$ $\begin{array}{lll}0.025 & 0.004 & 0.157\end{array}$ $\begin{array}{lll}0.010 & 0.003 & 0.030\end{array}$ $\begin{array}{lll}0.012 & 0.005 & 0.033\end{array}$ $\begin{array}{lll}0.012 & 0.005 & 0.033 \\ 0.005 & 0.001 & 0.036\end{array}$ $\begin{array}{lll}0.005 & 0.001 & 0.036 \\ 0.025 & 0.004 & 0.157\end{array}$ $\begin{array}{lll}0.011 & 0.003 & 0.045\end{array}$

\begin{tabular}{ll|l|l} 
& \multicolumn{2}{l}{ Ever } \\
Z-value & \multicolumn{1}{l}{$\boldsymbol{P}$-value } & & \\
-1.820 & 0.069 & & \\
-3.617 & 0.000 & & \\
-3.878 & 0.000 & & \\
-3.617 & 0.000 & & \\
-7.943 & 0.000 & & \\
-8.690 & 0.000 & & \\
-5.233 & 0.000 & & \\
-3.617 & 0.000 & & \\
-6.264 & 0.000 & & \\
& & -1.00 &
\end{tabular}

Event rate and $95 \% \mathrm{Cl}$

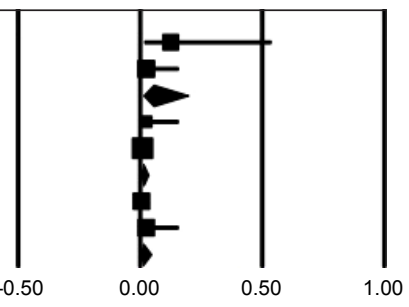

D

\begin{tabular}{lll}
$\begin{array}{l}\text { Group by } \\
\text { Subgroup }\end{array}$ & Study & $\begin{array}{l}\text { Adverse events } \\
\text { grade }>3\end{array}$ \\
within study & & \\
\hline
\end{tabular}

\section{Increased ALT}

Increased ALT

Increased ALT*

Increased AST

Increased AST

Increased AST*

\section{Powles et al, ${ }^{17} 2017 \quad$ Increased ALT}

Wainberg et al, ${ }^{11} 2017$ Increased ALT

Powles et al, ${ }^{17} 2017$

Increased AST

Increased AST
Meta analysis

Statistics for each study Event Lower Upper rate limit limit Z-value $P$-value

$\begin{array}{lllll}0.010 & 0.003 & 0.041 & -6.399 & 0.000\end{array}$

$\begin{array}{lllll}0.050 & 0.013 & 0.179 & -4.059 & 0.000\end{array}$

$\begin{array}{lllll}0.023 & 0.005 & 0.101 & -4.679 & 0.000\end{array}$

$\begin{array}{lllll}0.016 & 0.005 & 0.048 & -7.110 & 0.000\end{array}$

$\begin{array}{lllll}0.075 & 0.024 & 0.208 & -4.185 & 0.000\end{array}$

$\begin{array}{lllll}0.034 & 0.007 & 0.149 & -4.099 & 0.000\end{array}$
Event rate and $95 \% \mathrm{Cl}$

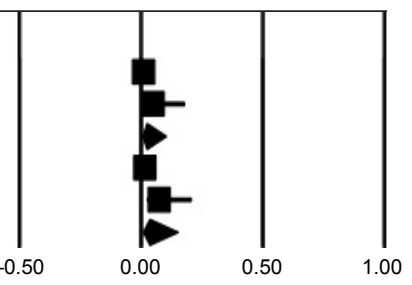

E

Group by

Subgroup within study

\section{Increased ALT}

Increased ALT

Increased ALT*

Increased AST

Increased AST

Increased AST*

Pruritus

Pruritus

Pruritus*

Study

Adverse events

Meta analysis

Statistics for each study

Event Lower Upper

rate limit limit Z-value P-value

$\begin{array}{lllll}0.175 & 0.086 & 0.324 & -3.726 & 0.000\end{array}$

$\begin{array}{lllll}0.101 & 0.055 & 0.178 & -6.554 & 0.000\end{array}$

$\begin{array}{lllll}0.126 & 0.080 & 0.193 & -7.445 & 0.000\end{array}$

Antonia et al, ${ }^{21} 2016 \quad$ Increased ALT

Kelley et al, ${ }^{10} 2017$

Increased AST

$\begin{array}{lll}0.150 & 0.069 & 0.296\end{array}$

$\begin{array}{lll}0.081 & 0.041 & 0.153\end{array}$

$\begin{array}{lll}0.081 & 0.041 & 0.153 \\ 0.105 & 0.063 & 0.169\end{array}$

$\begin{array}{lll}0.175 & 0.086 & 0.324\end{array}$

Kelley et al, ${ }^{10} 2017$

Pruritus

Antonia et al, ${ }^{21} 2016 \quad$ Pruritus

$\begin{array}{lll}0.212 & 0.143 \quad 0.304\end{array}$

$\begin{array}{lll}0.202 & 0.143 \quad 0.277\end{array}$

$-3.917 \quad 0.000$

$-6.593 \quad 0.000$

$-7.573 \quad 0.000$

$\begin{array}{ll}-3.726 & 0.000\end{array}$

$-5.337 \quad 0.000$

$-6.491$

0.000

Figure 2 The rates and $95 \% \mathrm{Cl}$ of major $\mathrm{AEs}$.

Notes: $\mathrm{AE}$ rates and $95 \% \mathrm{Cl}$ of $(\mathbf{A})$ a fixed model for durvalumab alone and $(\mathbf{B})$ a random model for durvalumab alone. Grade $\geq 3 \mathrm{AE}$ rates and $95 \% \mathrm{Cl}$ of $(\mathbf{C})$ a fixed model for durvalumab alone and (D) a random model for durvalumab alone. (E) AE rates and $95 \% \mathrm{Cl}$ of a fixed model for combination treatment with durvalumab and tremelimumab. *Analysis results of different trials for the adverse event.

Abbreviations: AE, adverse event; ALP, alkaline phosphatase; ALT, alanine transaminase; AST, aspartate aminotransferase.

compared with placebo (mPFS: $16.8 \%$ vs $5.6 \%$ ) and is now recommended in the National Comprehensive Cancer Network (NCCN) guidelines..$^{28} \mathrm{~A}$ large number of trials are currently ongoing, including the application of durvalumab in NSCLC, head and neck cancer, gastric cancer, hepatocellular carcinoma, pancreatic cancer, mesothelioma, and hematologic cancers. ${ }^{4,5}$

Durvalumab attacks cancer cells via a complex mechanism. Under normal physiological conditions, PD-L1 is commonly expressed on ICs, epithelial cells, and endothelial cells, and its overexpression is observed in various cancers. ${ }^{5}$ The single-chain variable domain of durvalumab interacts with the immunoglobulin variable domain of PD-L1 on cancer cells, causing a steric clash that hinders the binding of PD-1 to PD-L1 and leads to T-cell activation and proliferation..$^{29}$ Durvalumab has been demonstrated to inhibit tumor growth via a T-cell-associated mechanism in mouse xenograft models. ${ }^{30}$ The binding kinetics of durvalumab are similar to those of atezolizumab, which has been approved by the US FDA. ${ }^{5}$ Durvalumab does not bind with PD-L2 on macrophages and dendritic cells, avoiding potential toxic effects caused by PD-L2 inhibition. ${ }^{29}$

The recommended dosage of durvalumab is $10 \mathrm{mg} / \mathrm{kg}$ every 2 weeks via intravenous infusion until progression or unacceptable toxicity. ${ }^{27}$ Durvalumab exhibits a manageable 
Table 2 Adverse events leading to therapy discontinuation

\begin{tabular}{|c|c|c|c|c|}
\hline $\begin{array}{l}\text { Clinical trial } \\
\text { information }\end{array}$ & Serious AEs & Incidence & Therapy & $\begin{array}{l}\text { Cancer } \\
\text { type }\end{array}$ \\
\hline NCT019386I2 & Grade 2 pneumonitis & $1 / 22$ & Durvalumab & Solid tumors \\
\hline NCT02। 4 I347 & NM & $1 / 8$ & Durvalumab & Solid tumors \\
\hline NCT02087423 & NM & $9 / 333$ & Durvalumab & NSCLC \\
\hline \multirow[t]{3}{*}{ NCT0I693562 } & Autoimmune hepatitis & $1 / 191$ & Durvalumab & UCC \\
\hline & Pneumonitis & $1 / 191$ & & \\
\hline & NM & $1 / 191$ & & \\
\hline \multirow[t]{3}{*}{ NCT025I 9348} & Grade 3 pneumonitis & $1 / 40$ & Durvalumab & $\mathrm{HCC}$ \\
\hline & Grade 3 colitis/diarrhea & $1 / 40$ & & \\
\hline & Asymptomatic grade 4 elevated AST and ALT & $1 / 40$ & & \\
\hline \multirow[t]{4}{*}{ NCT0I 693562} & Grade I-2 pneumonitis & $5 / 304$ & Durvalumab & NSCLC \\
\hline & Grade 4 pneumonitis & $1 / 304$ & & \\
\hline & Grade $2-3$ colitis & $4 / 304$ & & \\
\hline & Grade 4 colitis & $1 / 304$ & & \\
\hline \multirow[t]{2}{*}{ NCT0202796I } & Grade 3 thrombocytopenia & $1 / 50$ & Durvalumab + trametinib \pm & Melanoma \\
\hline & Grade 3 choroidal effusion & $1 / 50$ & dabrafenib & \\
\hline NCT02I 43466 & Grade 3-4 ILD & $5 / 34$ & Durvalumab + AZD929I & NSCLC \\
\hline \multirow[t]{2}{*}{ NCT02088II 2} & Grade 3-4 increased ALT and/or AST & $3 / 20$ & Durvalumab + gefitinib & NSCLC \\
\hline & Grade 3-4 pneumonitis & $1 / 20$ & & \\
\hline \multirow[t]{4}{*}{ NCT02000947 } & Colitis & $9 / 102$ & Durvalumab + tremelimumab & NSCLC \\
\hline & Diarrhea & $5 / 102$ & & \\
\hline & Pneumonitis & $5 / 102$ & & \\
\hline & NM & $10 / 102$ & & \\
\hline
\end{tabular}

Abbreviations: AEs, adverse events; ALT, alanine transaminase; AST, aspartate aminotransferase; HCC, hepatocellular carcinoma; ILD, interstitial lung disease; NM, not mentioned; NSCLC, non-small-cell lung cancer; UCC, urothelial carcinoma.

safety profile and early-stage antitumor activity in various cancers, particularly those that are PD-L1-positive.

Our analysis indicates that the most common AEs associated with durvalumab treatment are pruritus and fatigue. This result is consistent with previous reports on other PD-1/ PD-L1 inhibitors; pruritus, a dermatological AE, is observed in $34 \%-39 \%$ of patients and fatigue in $12 \%-37 \%$ of patients receiving PD-1/PD-L1 inhibitors. ${ }^{31}$ In our study, durvalumab was associated with a similar incidence of fatigue and a lower incidence of pruritus. Fortunately, the AEs mentioned above are generally mild and not dose-related. ${ }^{32}$

As a PD-L1 inhibitor, durvalumab not only helps to inhibit cancer development but also induces various immune responses. Such immune-related adverse events (irAEs)

Table 3 Efficacy of treatment with durvalumab alone

\begin{tabular}{|c|c|c|c|c|c|c|c|c|c|}
\hline $\begin{array}{l}\text { Clinical trial } \\
\text { information }\end{array}$ & \multicolumn{2}{|l|}{ Subgroup } & $\begin{array}{l}\text { Evaluable } \\
\text { patients }(\mathrm{N})\end{array}$ & $\begin{array}{l}\text { PR } \\
(\mathrm{N})\end{array}$ & $\begin{array}{l}\text { CR } \\
(\mathrm{N})\end{array}$ & $\begin{array}{l}\text { SD } \\
(\mathrm{N})\end{array}$ & $\begin{array}{l}\text { ORR \% } \\
(95 \% \mathrm{Cl})\end{array}$ & $\begin{array}{l}\text { mPFS months } \\
(95 \% \mathrm{Cl})\end{array}$ & $\begin{array}{l}\text { mOS months } \\
(95 \% \mathrm{Cl})\end{array}$ \\
\hline NCT019386I2 & \multicolumn{2}{|l|}{ Solid tumors } & 22 & I & NM & 6 & NM & NM & NM \\
\hline \multirow[t]{3}{*}{ NCT02087423 } & \multirow[t]{2}{*}{ Cohort 2} & $\begin{array}{l}\text { PD-LI } \geq 25 \% \\
\text { of } T C s\end{array}$ & 146 & NM & NM & NM & $16.4(10.8-23.5)$ & $3.3(1.9-3.7)$ & $10.9(8.6-13.6)$ \\
\hline & & $\begin{array}{l}\text { PD-LI }<25 \% \\
\text { of TCs }\end{array}$ & 53 & NM & NM & NM & $7.5(3.1-14.9)$ & $1.9(1.8-1.9)$ & $9.3(5.9-10.8)$ \\
\hline & Cohort 3 & $\begin{array}{l}\text { PD-LI } \geq 90 \% \\
\text { of } T C s\end{array}$ & 68 & NM & NM & NM & $30.9(20.2-43.3)$ & $2.4(1.8-5.5)$ & NR (5.9-NE) \\
\hline \multirow[t]{2}{*}{ NCT0I693562 } & \multirow[t]{2}{*}{ UCC } & PD-LI high & 98 & 23 & 4 & NM & $27.6(19.0-37.5)$ & $2.1(1.4-2.8)$ & 20.0 (II.6-NE) \\
\hline & & $\begin{array}{l}\text { PD-LI } \\
\text { low/negative }\end{array}$ & 79 & 2 & 2 & NM & $5.1(1.4-12.5)$ & $1.4(1.3-1.5)$ & 8.1 (3.I-NE) \\
\hline NCTOI 693562 & \multicolumn{2}{|l|}{$\mathrm{HCC}$} & 30 & 4 & NM & 14 & $10.0(2.8-23.7)$ & $2.7(1.4-5.3)$ & I3.2(6.3-2|.I) \\
\hline NCT02336I65 & Glioblastoma & Cohort B & 30 & 4 & NM & 14 & NM & NM & NM \\
\hline
\end{tabular}

Notes: PD-LI high, $\geq 25 \%$ of either tumor cells or immune cells expressing PD-LI; PD-LI low or negative, $<25 \%$ of both tumor cells and immune cells expressing PD-LI. Abbreviations: CR, complete response; HCC, hepatocellular carcinoma; m, median; mOS, median overall survival; mPFS, median progression-free survival; NE, not estimated; NM, not mentioned; NR, not reached; ORR, objective response rate; OS, overall survival; PD-LI, programmed cell death ligand-I; PFS, progression-free survival; $\mathrm{PR}$, partial response; SD, stable disease; TCs, tumor cells; UCC, urothelial carcinoma. 
Table 4 The efficacy of durvalumab and tremelimumab

\begin{tabular}{lllll}
\hline $\begin{array}{l}\text { Clinical trial } \\
\text { information }\end{array}$ & Subgroup & $\begin{array}{l}\text { Evaluable } \\
\text { patient }\end{array}$ & $\begin{array}{l}\text { PR } \\
\text { (N) }\end{array}$ & $\begin{array}{l}\text { ORR } \% \\
(\mathbf{9 5} \% \mathbf{C l})\end{array}$ \\
\hline NCT02536794 & Breast cancer & 18 & 3 & 17 \\
NCT02519348 & HCC & 40 & NM & 15 \\
NCT0197583I & Cervical cancer & 13 & 0 & NM \\
& Colorectal cancer & 11 & I & NM \\
& NTNBC & 10 & I & NM \\
& Ovarian cancer & 25 & 2 & NM \\
& RCC & 11 & I & NM \\
NCT02000947 & PD-LI-positive NSCLC & 18 & NM $33(13-59)$ \\
& PD-LI-negative NSCLC & 37 & NM $30(16-47)$ \\
& PD-LI 0\% NSCLC & 24 & NM $38(19-59)$ \\
\hline
\end{tabular}

Notes: PD-LI negative, $<25 \%$ but $<0 \%$ of tumor cells expressing PD-LI; PD-LI positive, $\geq 25 \%$ of tumor cells expressing PD-LI.

Abbreviations: HCC, hepatocellular carcinoma; NM, not mentioned; NSCLC, nonsmall-cell lung cancer; NTNBC, non-triple-negative breast cancer; ORR, objective response rate; PD-LI, programmed cell death ligand-I; PR, partial response; RCC, renal cell carcinoma.

can influence many systems, causing diarrhea, colitis, skin rash, hyperthyroidism, hypothyroidism, thyroiditis, hepatic toxicities, pneumonitis, and other rare toxicities. ${ }^{33}$ Among these, skin rash is the most common, occurring in $34 \%$ patients receiving nivolumab and $39 \%$ of those administered pembrolizumab for melanoma. ${ }^{34,35}$ According to our study, the incidence of skin rash in patients receiving durvalumab is lower. Damage of the pulmonary system presents as pneumonitis of the lung parenchyma and occurs in $<10 \%$ of patients treated with PD-1/PD-L1 inhibitors..$^{33}$ Our analyses indicate that the occurrence of pneumonitis is rare among patients treated with durvalumab; therefore, compared with other PD-1/PD-L1 inhibitors, durvalumab is safer and its associated AEs are more tolerable.

To alleviate irAEs, immunosuppressive agents, including corticosteroids, antihistamines, and antitumor necrosis factor, can be used temporarily, without eliminating the antitumor response. ${ }^{36}$ Intravenous corticosteroids should be the first choice, while other immunosuppressive drugs, such as infliximab, could also be considered if corticosteroids are ineffective. ${ }^{36}$

The correlation between PD-L1 expression and efficacy remains a topic of discussion, and definitions of PD-L1 expression levels and the cutoff values used are controversial. Higher pretreatment PD-L1 expression $(\geq 25 \%)$ and detectable interferon-gamma mRNA expression in tumor biopsies were reported as associated with higher ORR and better overall survival (OS) in NSCLC patients. ${ }^{37}$ Subsequently, Massard et al reported that PD-L1 status, defined by TC or IC (positive: TC or IC $\geq 25 \%$; negative: TC and IC $<25 \%$ ), was not associated with durvalumab efficacy; rather, the combination of TC and IC had predictive value..$^{38}$ Furthermore, agreement is yet to be reached on the standard antibody for testing PD-L1 expression by immunohistochemistry (IHC). The Blueprint PD-L1 IHC Assay Comparison Project compared four PD-L1 IHC assays (22C3, 28-8, SP263, and SP142) and concluded that three of them $(22 \mathrm{C} 3,28-8$, and SP263) generated similar TC staining; however, analysis of IC staining was lacking. ${ }^{39}$ PD-L1 expression varies among different tumor types and may alter following treatment; ${ }^{40}$ therefore, the expression of PD-L1 should be tested in samples obtained at several time points or from different locations.

PD-1/PD-L1 inhibitors are among the most effective immunotherapy methods; however, even for melanoma, the type of cancer most sensitive to immunotherapy, $60 \%$ patients continue to display primary resistance. ${ }^{5}$ Primary resistance is related to numerous factors, including both those that are tumor intrinsic and those that are extrinsic. ${ }^{41}$ Mutational burden is one of the most significant tumor intrinsic factors. ${ }^{42}$ Cancers with higher mutational loads, such as melanoma and NSCLC, exhibit superior responses to immunotherapy ${ }^{43}$ For a given type of cancer, patients with higher nonsynonymous mutation burdens will have improved treatment responses and longer progression-free survival (PFS) ${ }^{44} E G F R$ is one of the most commonly mutated oncogenes in NSCLC. Patients with NSCLC with EGFR mutations or anaplastic lymphoma kinase $(A L K)$ rearrangements exhibit limited responses to nivolumab, pembrolizumab, or atezolizumab. ${ }^{45}$ For NSCLC patients treated with PD-1/PD-L1 inhibitors, the objective responses were only $3.6 \%(1 / 28)$ in EGFR-mutant or $A L K$-positive patients vs $23.3 \%$ (7/30) in patients negative for these markers. ${ }^{45}$ Moreover, $\sim 4$ weeks after initial PR, these patients subsequently progressed. The latest update of ATLANTIC (NCT02087423) also demonstrated that the response was superior in $\mathrm{EGFR}^{-} / \mathrm{ALK}^{-}$NSCLC patients treated with durvalumab. ${ }^{46}$ Resistance may be associated with oncogenic signals in patients with $E G F R$ mutations and $A L K$ rearrangements, causing immunosuppression. ${ }^{47,48}$ Although EGFR mutations and $A L K$ rearrangements lead to elevated PD-L1 expression, its levels are reduced after treatment with $E G F R$ or $A L K$ tyrosine kinase inhibitors (TKIs), which could cause resistance to PD-1/PD-L1 inhibitors. ${ }^{47-49}$ The major extrinsic factors influencing primary resistance are immunoregulatory factors within the tumor microenvironment, such as low PD-L1 expression levels, insufficient numbers of tumor-infiltrating lymphocytes, and severe exhaustion of $\mathrm{T}$ cells. ${ }^{41,42}$ The majority of primary responders eventually developed acquired resistance after treatment. The mechanism underlying such resistance may be associated with the 
loss of T-cell functional phenotype, as well as mutations of Janus kinase $(J A K 1 / 2)$, which limit the presentation of tumor antigens. ${ }^{42,50}$

To optimize the use of durvalumab and overcome resistance, combinations with other therapies have been designed. The combination of durvalumab with radiotherapy or chemotherapy resulted in IC death and the release of tumor antigens to promote immune responses. ${ }^{28,51}$ Inhibition of immunosuppressive factors can also enhance antitumor immunity. ${ }^{21,52}$ Among these, combination treatment with anti-CTLA-4 antibodies is particularly noteworthy because CTLA-4/B7 interaction has a separate function in T-cell immunity and could be used to target PD-L1-negative tumors. Patients with PD-L1-positive or -negative NSCLC who received durvalumab and tremelimumab combination therapy had similar ORRs because of the inclusion of the anti-CTLA-4 antibody. ${ }^{21}$ Our study indicates that the incidence of AEs increases but remains tolerable when combining durvalumab with other types of immunotherapy. Given tolerable AEs, the ORRs of combination immunotherapies are of great significance for cancer treatment.

Lung cancer was the major type of malignancy focused on among completed and ongoing trials of durvalumab, alone or as combination therapy with durvalumab and

Table 5 Functions of durvalumab in ongoing trials of lung cancer

\begin{tabular}{|c|c|c|c|}
\hline $\begin{array}{l}\text { Clinical trial } \\
\text { information }\end{array}$ & Phase & Therapy & Disease \\
\hline \multicolumn{4}{|c|}{ Maintenance therapy } \\
\hline NCT02।I7I67 & II & Durvalumab & $\begin{array}{l}\text { Metastatic NSCLC and SD/PR after four cycles of an induction } \\
\text { platinum-based chemotherapy }\end{array}$ \\
\hline NCT02I2546I & III & Durvalumab & $\begin{array}{l}\text { Stage III unresectable NSCLC and not progressed following } \\
\text { definitive, platinum-based, concurrent chemoradiotherapy }\end{array}$ \\
\hline \multicolumn{4}{|c|}{ Neoadjuvant and adjuvant therapy } \\
\hline NCT02273375 & III & Durvalumab & Completely resected NSCLC \\
\hline NCT02572843 & II & Durvalumab & Primary resectable stage IIIA (N2) NSCLC \\
\hline NCT03030I3I & II & Durvalumab & Early stage (I-IIIA) NSCLC \\
\hline \multicolumn{4}{|l|}{ First-line therapy } \\
\hline NCT028796I7 & II & Durvalumab & Advanced NSCLC with ECOG performance status of 2 \\
\hline NCT03003962 & III & Durvalumab & $\begin{array}{l}\text { Advanced NSCLC with EGFR and ALK wild-type and high } \\
\text { expression of PD-LI }\end{array}$ \\
\hline NCT03I646I6 & III & $\begin{array}{l}\text { Durvalumab }+ \text { chemotherapy with/ } \\
\text { without tremelimumab }\end{array}$ & Metastatic NSCLC with EGFR and ALK wild-type \\
\hline NCT02453282 & III & $\begin{array}{l}\text { Durvalumab with/without } \\
\text { tremelimumab }\end{array}$ & NSCLC \\
\hline NCT02542293 & III & Durvalumab + tremelimumab & NSCLC \\
\hline \multicolumn{4}{|c|}{ With radiation therapy } \\
\hline NCT02888743 & II & $\begin{array}{l}\text { Durvalumab + tremelimumab with/ } \\
\text { without RT }\end{array}$ & Stage IV NSCLC \\
\hline NCT03275597 & I & Durvalumab + tremelimumab + SBRT & Stage IV oligometastatic NSCLC with EGFR and ALK wild-type \\
\hline \multicolumn{4}{|l|}{ Others } \\
\hline NCT026699/4 & II & Durvalumab & Lung cancer with refractory/recurrent brain metastases \\
\hline NCT02352948 & III & $\begin{array}{l}\text { Durvalumab with/without } \\
\text { tremelimumab }\end{array}$ & $\begin{array}{l}\text { Locally advanced or metastatic NSCLC (stage IIIB-IV) with } \\
\text { EGFR and ALK wild-type }\end{array}$ \\
\hline NCT0240327I & $1 / / 1$ & Durvalumab + ibrutinib & Relapsed or refractory NSCLC \\
\hline NCT02503774 & I & Durvalumab + MEDI9447 & Advanced lung cancer \\
\hline NCT02740985 & I & Durvalumab + AZD4635 & Advanced or metastatic NSCLC with EGFR and ALK wild-type \\
\hline NCT02805660 & $1 / I I$ & Durvalumab + mocetinostat & Advanced or metastatic NSCLC \\
\hline NCT02898II6 & $1 / / 1$ & Durvalumab + ensartinib & ALK-rearranged NSCLC \\
\hline NCT02983578 & II & Durvalumab + AZD9150 & Advanced NSCLC \\
\hline NCT03। 64772 & $\mathrm{I} / \mathrm{ll}$ & $\begin{array}{l}\text { Durvalumab + mRNA vaccine with/ } \\
\text { without tremelimumab }\end{array}$ & Metastatic NSCLC with ALK wild-type \\
\hline NCT033346I7 & II & $\begin{array}{l}\text { Durvalumab + olaparib/AZD9150/ } \\
\text { AZD6738/vistusertib }\end{array}$ & $\begin{array}{l}\text { Metastatic or recurrent NSCLC with EGFR/ALK/ROS-I } \\
\text { wild-type and progressed on an anti-PD-I/PD-LI containing } \\
\text { therapy }\end{array}$ \\
\hline
\end{tabular}

Abbreviations: ALK, anaplastic lymphoma kinase; ECOG, Eastern Cooperative Oncology Group; EGFR, epidermal growth factor receptor; NSCLC, non-small-cell lung cancer; PD-I, programmed cell death-I; PD-LI, programmed cell death ligand-I; PR, partial response; ROS-I, ROS proto-oncogene I, receptor tyrosine kinase; RT, radiation therapy; SBRT, stereotactic body radiotherapy; SD, stable disease. 
other treatments. A study on patients with NSCLC indicated that superior ORR, PFS, and OS were achieved using durvalumab to treat patients with high PD-L1 expression. ${ }^{16}$ In the trials discussed above, durvalumab was not only used alone but also combined with a CTLA-4 inhibitor (tremelimumab) for the treatment of advanced NSCLC or with EGFR-TKIs (gefitinib or AZD9291) for EGFR-positive NSCLC. ${ }^{21,22}$ In other ongoing or recruiting trials of NSCLC, durvalumab is reported to have multiple and noteworthy applications; for example, in maintenance, neoadjuvant/adjuvant, or firstline therapies. ${ }^{28,53-61}$ Several trials of the PD-L1 inhibitors, nivolumab and pembrolizumab, as neoadjuvant or adjuvant therapy are also in progress. ${ }^{62-65}$ Pembrolizumab was the first PD-L1 inhibitor approved by the US FDA as the first-line therapy for metastatic NSCLC patients with high PD-L1 expression ( $\geq 50 \%$ ) and $E G F R$ and $A L K$ wild-type, or in combination with pemetrexed and carboplatin for metastatic nonsquamous NSCLC. ${ }^{66}$ Similar trials are investigating durvalumab, and another breakthrough for first-line therapy is anticipated in the future. There are two trials investigating the combination of durvalumab, tremelimumab, and radiation therapy (RT). ${ }^{67,68}$ Because of the complicated effects of RT on the immune system, such combinations may lead to improved efficacy. Nevertheless, there are concerns about the damage to the pulmonary system and the incidence of ILD. HUDSON (NCT03334617) is another important trial focusing on the treatment after resistance to durvalumab and may generate feasible suggestions for clinical applications (Table 5) ${ }^{69}$

Overall, based on this meta-analysis of safety and efficacy, we conclude that durvalumab can be safely used for the treatment of many solid cancers and that its use in combination with tremelimumab deserves additional study. Combination treatment with durvalumab and tremelimumab leads to an increased incidence of AEs, including ALT, AST, and pruritus; therefore, patients receiving combination therapy should be closely followed up. High PD-L1 expression is a biomarker for better ORR, mPFS, and mOS for patients treated with durvalumab. Meanwhile, patients with high mutation burdens, EGFR wild-type, or $A L K$ rearrangement-negative tumors will achieve greater benefit from durvalumab. The numerous ongoing or recruiting trials evaluating durvalumab and combination therapies for different solid tumors will provide additional data in the future.

\section{Acknowledgment}

We express our gratitude to Miss Sha Zhu who provided a great deal of assistance with manuscript editing, along with meaningful advice.

\section{Disclosure}

The authors report no conflicts of interest in this work.

\section{References}

1. Siegel RL, Miller KD, Jemal A. Cancer Statistics, 2017. CA Cancer J Clin. 2017;67(1):7-30.

2. Miller KD, Siegel RL, Lin CC, et al. Cancer treatment and survivorship statistics, 2016. CA Cancer J Clin. 2016;66(4):271-289.

3. Bellmunt J, Powles T, Vogelzang NJ. A review on the evolution of PD-1/PD-L1 immunotherapy for bladder cancer: the future is now. Cancer Treat Rev. 2017;54:58-67.

4. Sharma P, Allison JP. The future of immune checkpoint therapy. Science. 2015;348(6230):56-61.

5. Zou W, Wolchok JD, Chen L. PD-L1 (B7-H1) and PD-1 pathway blockade for cancer therapy: mechanisms, response biomarkers, and combinations. Sci Transl Med. 2016;8(328):328rv4.

6. Freeman GJ, Long AJ, Iwai Y, et al. Engagement of the PD-1 immunoinhibitory receptor by a novel B7 family member leads to negative regulation of lymphocyte activation. J Exp Med. 2000;192(7):1027-1034.

7. Schildberg FA, Klein SR, Freeman GJ, Sharpe AH. Coinhibitory pathways in the B7-CD28 ligand-receptor family. Immunity. 2016;44(5): 955-972.

8. Hodi FS, O'Day SJ, McDermott DF, et al. Improved survival with ipilimumab in patients with metastatic melanoma. N Engl J Med. 2010; 363(8):711-723.

9. Ibrahim R, Stewart R, Shalabi A. PD-L1 blockade for cancer treatment: MEDI4736. Semin Oncol. 2015;42(3):474-483.

10. Kelley RK, Abou-Alfa GK, Bendell JC, et al. Phase I/II study of durvalumab and tremelimumab in patients with unresectable hepatocellular carcinoma (HCC): Phase I safety and efficacy analyses. J Clin Oncol. 2017;35(15):4073.

11. Wainberg ZA, Segal NH, Jaeger D, et al. Safety and clinical activity of durvalumab monotherapy in patients with hepatocellular carcinoma (HCC). J Clin Oncol. 2017;35(15):4071.

12. Reardon DA, Kaley TJ, Dietrich J, et al. Phase 2 study to evaluate safety and efficacy of MEDI4736 (durvalumab [DUR]) in glioblastoma (GBM) patients: an update. J Clin Oncol. 2017;35(15):2042.

13. Lin CC, Golan T, Corral J, et al. Phase 1 study of ramucirumab (R) plus durvalumab (D) in patients (pts) with locally advanced and unresectable or metastatic gastrointestinal or thoracic malignancies (NCT02572687); Phase 1a results. Ann Oncol. 2016;27(Suppl 8):viii1-viii17.

14. Antonia SJ, Kim SW, Spira AI, et al. Safety and clinical activity of durvalumab (MEDI4736), an anti-PD-LI antibody, in treatment-naive patients with advanced non-small-cell lung cancer. J Clin Oncol. 2016; 34:9029.

15. Segal NH, Ou SHI, Balmanoukian AS, et al. Updated safety and efficacy of durvalumab (MEDI4736), an anti-PD-L 1 antibody, in patients from a squamous cell carcinoma of the head and neck (SCCHN) expansion cohort. Ann Oncol. 2016;27(Suppl 6):vi328-vi350.

16. Garassino M, Vansteenkiste J, Kim JH, et al. PL04a.03: durvalumab in $\geq 3$ rd-line locally advanced or metastatic, EGFR/ALK wild-type NSCLC: results from the phase 2 ATLANTIC study. JClin Oncol. 2017; 12(1):S10-S11.

17. Powles T, O'Donnell PH, Massard C, et al. Efficacy and safety of Durvalumab in locally advanced or metastatic urothelial carcinoma: updated results from a phase 1/2 open-label study. JAMA Oncol. 2017; 3(9):e172411.

18. Callahan MK, Odunsi K, Sznol M, et al. Phase 1 study to evaluate the safety and tolerability of MEDI4736 (durvalumab, DUR) + tremelimumab (TRE) in patients with advanced solid tumors. $J$ Clin Oncol. 2017;35(15):3069.

19. Takahashi Y, Fujikawa K, Sagawa T, et al. A phase 1 study to assess the safety and tolerability of tremelimumab alone and in combination with MEDI4736 in Japanese patients with advanced solid malignancies. Eur J Cancer. 2015;5:S107. 
20. Ahn MJ, Sun JM, Lee SH, Ahn JS, Park K. EGFR TKI combination with immunotherapy in non-small cell lung cancer. Expert Opin Drug Saf. 2017;16(4):465-469.

21. Antonia S, Goldberg SB, Balmanoukian A, et al. Safety and antitumour activity of durvalumab plus tremelimumab in non-small cell lung cancer: a multicenter, phase 1b study. Lancet Oncol. 2016;17(3):299-308.

22. Gibbons DL, Chow LQ, Kim DW, et al. 570 Efficacy, safety and tolerability of MEDI4736 (durvalumab [D]), a human IgG1 antiprogrammed cell death-ligand-1 (PD-L1) antibody, combined with gefitinib (G): a phase I expansion in TKI-naïve patients (pts) with EGFR mutant NSCLC. J Thorac Oncol. 2016;11(Suppl 4):S79.

23. Lee JM, Cimino-Mathews A, Peer CJ, et al. Safety and clinical activity of the programmed death-ligand 1 inhibitor durvalumab in combination with poly (ADP-ribose) polymerase inhibitor olaparib or vascular endothelial growth factor receptor 1-3 inhibitor cediranib in women's cancers: a dose-escalation, phase I study. J Clin Oncol. 2017;35(19): 2193-2202.

24. Ribas A, Butler M, Lutzky J, et al. Phase I study combining antiPD-L1 (MEDI4736) with BRAF (dabrafenib) and:or MEK (trametinib) inhibitors in advanced melanoma. J Clin Oncol. 2015;33(Suppl 15): 3003.

25. Iguchi H, Nogami N, Kozuki T, et al. Phase I study to evaluate the safety and tolerability of MEDI4736, an anti-programmed cell death ligand-1 (PD-L1) antibody, in Japanese patients with advanced solid tumors. J Clin Oncol. 2015;33(Suppl 15):3039.

26. Santa-Maria CA, Jain S, Flaum L, et al. A phase II study of PD-L1 and CTLA-4 inhibition and immunopharmcogenomics in metastatic breast cancer. Cancer Res. 2017;77(Suppl 4):OT3-01-01.

27. Imfinzi ${ }^{\mathrm{TM}}$ (durvalumab): US prescribing information. 2017. Available from: https://www.fda.gov. Accessed May 24, 2017.

28. Antonia SJ, Villegas A, Daniel D, et al. Durvalumab after chemoradiotherapy in stage III non-small-cell lung cancer. $N$ Engl J Med. 2017; 377(20):1919-1929.

29. Zak KM, Kitel R, Przetocka S, et al. Structure of the complex of human programmed death 1, PD-1, and its ligand PD-L1. Structure. 2015;23(12): 2341-2348.

30. Syed YY. Durvalumab: first global approval. Drugs. 2017;77(12): 1369-1376.

31. Naidoo J, Page DB, Li BT, et al. Toxicities of the anti-PD-1 and anti-PD-L1 immune checkpoint antibodies. Ann Oncol. 2015;26(12): 2375-2391.

32. Cousin S, Seneschal J, Italiano A. Toxicity profiles of immunotherapy. Pharmacol Ther. 2018;181:91-100.

33. Weber JS, Yang JC, Atkins MB, Disis ML. Toxicities of immunotherapy for the practitioner. J Clin Oncol. 2015;33(18):2092-2099.

34. Weber JS, Antonia SJ, Topalian SL, et al. Safety profile of nivolumab (NIVO) in patients (pts) with advanced melanoma (MEL): a pooled analysis. J Clin Oncol. 2015;33(Suppl 15):9018.

35. Robert C, Schachter J, Long GV, et al. Pembrolizumab versus ipilimumab in advanced melanoma. $N$ Engl J Med. 2015;372(26):2521-2532.

36. Horvat TZ, Adel NG, Dang TO, et al. Immune-related adverse events, need for systemic immunosuppression, and effects on survival and time to treatment failure in patients with melanoma treated with ipilimumab at Memorial Sloan Kettering Cancer Center. J Clin Oncol. 2015;33(28): 3193-3198.

37. Higgs BW, Morehouse C, Streicher K, et al. Relationship of baseline tumoral IFNc mRNA and PD-L1 protein expression to overall survival in durvalumab-treated NSCLC patients. J Clin Oncol. 2016; 34(Suppl):3036.

38. Massard C, Gordon MS, Sharma S, et al. Safety and efficacy of Durvalumab(MEDI4736), an anti-programmed cell death ligand-1 immune checkpoint inhibitor, in patients with advanced urothelial bladder cancer. J Clin Oncol. 2016;34(26):3119-3125.

39. Hirsch FR, McElhinny A, Stanforth D, et al. PD-L1 immunohistochemistry assays for lung cancer: results from phase 1 of the Blueprint PD-L1 IHC Assay Comparison Project. J Thorac Oncol. 2017;12(2): 208-222.
40. Madore J, Vilain RE, Menzies AM, et al. PD-L1 expression in melanoma shows marked heterogeneity within and between patients: implications for anti-PD-1/PD-L1 clinical trials. Pigment Cell Melanoma Res. 2015;28(3):245-253.

41. Pitt JM, Vétizou M, Daillère R, et al. Resistance mechanisms to immunecheckpoint blockade in cancer: tumor-intrinsic and-extrinsic factors. Immunity. 2016;44(6):1255-1269.

42. Wang Q, Wu X. Primary and acquired resistance to PD-1/PD-L1 blockade in cancer treatment. Int Immunopharmacol. 2017;46:210-219.

43. Topalian SL, Hodi FS, Brahmer JR, et al. Safety, activity, and immune correlates of anti-PD-1 antibody in cancer. NEngl J Med. 2012;366(26): 2443-2454.

44. Rizvi NA, Hellmann MD, Snyder KA, et al. Mutational landscape determines sensitivity to PD-1 blockade in non-small cell lung cancer. Science. 2015;348(6230):124-128.

45. Gainor JF, Shaw AT, Sequist LV, et al. EGFR mutations and ALK rearrangements are associated with low response rates to PD-1 pathway blockade in non-small cell lung cancer: a retrospective analysis. Clin Cancer Res. 2016;22(18):4585-4593.

46. Garassino MC, Cho BC, Kim JH, et al; ATLANTIC Investigators. Durvalumab as third-line or later treatment for advanced non-small-cell lung cancer (ATLANTIC): an open-label, single-arm, phase 2 study. Lancet Oncol. 2018;19(4):521-536.

47. Akbay EA, Koyama S, Carretero J, et al. Activation of the PD-1 pathway contributes to immune escape in EGFR-driven lung tumors. Cancer Discov. 2013;3(12):1355-1363.

48. Ota K, Azuma K, Kawahara A, et al. Induction of PD-L1 expression by the EML4-ALK oncoprotein and downstream signaling pathways in non-small cell lung cancer. Clin Cancer Res. 2015;21(17): 4014-4021.

49. Zhang M, Li G, Wang Y, et al. PD-L1 expression in lung cancer and its correlation with driver mutations: a meta-analysis. Sci Rep. 2017; $7(1): 10255$.

50. Sharma P, Hu-Lieskovan S, Wargo JA, Ribas A. Primary, adaptive, and acquired resistance to cancer immunotherapy. Cell. 2017;168(4): 707-723.

51. Twyman-Saint Victor C, Rech AJ, Maity A, et al. Radiation and dual checkpoint blockade activate non-redundant immune mechanisms in cancer. Nature. 2015;520(7547):373-377.

52. Wolchok JD, Saenger Y. The mechanism of anti-CTLA-4 activity and the negative regulation of T-cell activation. Oncologist. 2008; 13(Suppl 4):2-9.

53. SAFIR02_Lung - Efficacy of targeted drugs guided by genomic profiles in metastatic NSCLC patients. Available from: https://ClinicalTrials. gov/show/NCT02117167. Accessed June 16, 2018.

54. Double blind placebo controlled study of adjuvant MEDI4736 In completely resected NSCLC. Available from: https://ClinicalTrials. gov/show/NCT02273375. Accessed June 16, 2018.

55. Anti-PD-L1 in stage IIIA(N2) non-small cell lung cancer (NSCLC). Available from: https://ClinicalTrials.gov/show/NCT02572843

56. Immune neoadjuvant therapy study of durvalumab in early stage nonsmall cell lung cancer. Available from: https://ClinicalTrials.gov/show/ NCT03030131. Accessed June 16, 2018.

57. A clinical trial of durvalumab (MEDI4736) as 1st line therapy in advanced non-small cell lung cancer patients. Available from: https:// ClinicalTrials.gov/show/NCT02879617. Accessed June 16, 2018.

58. A study of durvalumab versus standard of care in advanced non smallcell lung cancer. Available from: https://ClinicalTrials.gov/show/ NCT03003962. Accessed June 16, 2018.

59. Study of durvalumab + tremelimumab with chemotherapy or durvalumab with chemotherapy or chemotherapy alone for patients with lung cancer (POSEIDON). Available from: https://ClinicalTrials.gov/ show/NCT03164616. Accessed June 16, 2018.

60. Phase III open label first line therapy study of MEDI 4736 (durvalumab) with or without tremelimumab versus SOC in non small-cell lung cancer (NSCLC). Available from: https://ClinicalTrials.gov/show/ NCT02453282. Accessed June 16, 2018. 
61. Study of 1 st line therapy study of durvalumab with tremelimumab versus SoC in non small-cell lung cancer (NSCLC) (NEPTUNE). Available from: https://ClinicalTrials.gov/show/NCT02542293. Accessed June 16, 2018.

62. Study of induction checkpoint blockade for untreated stage I-IIIA nonsmall cell lung cancers amenable for surgical resection. Available from: https://ClinicalTrials.gov/show/NCT03158129. Accessed June 16, 2018.

63. Neoadjuvant anti PD-1 immunotherapy in resectable non-small cell lung cancer (NEOMUN). Available from: https://ClinicalTrials.gov/ show/NCT03197467. Accessed June 16, 2018.

64. Adjuvant pembrolizumab in N2 positive non-small Cell lung Cancer Patients. Available from: https://ClinicalTrials.gov/show/ NCT03053856. Accessed June 16, 2018.

65. Nivolumab after surgery and chemotherapy in treating patients with stage IB-IIIA non-small cell lung cancer (ANVIL). Available from: https://ClinicalTrials.gov/show/NCT02595944. Accessed June 16, 2018.
66. KEYTRUDA ${ }^{\circledR}$ (pembrolizumab). Full Prescribing Information. Whitehouse Station, NJ, USA: Merck \& Co., Inc; 2017.

67. Durvalumab and tremelimumab with or without high or low-dose radiation therapy in treating patients with metastatic colorectal or nonsmall cell lung cancer. Available from: https://ClinicalTrials.gov/show/ NCT02888743. Accessed June 16, 2018.

68. Phase Ib Study of Stereotactic Body Radiotherapy (SBRT) in Oligometastatic Non-small Lung Cancer (NSCLC) With Dual Immune Checkpoint Inhibition. Available from: https://ClinicalTrials.gov/show/ NCT03275597. Accessed June 16, 2018.

69. Phase II umbrella study of novel anti-cancer agents in patients with NSCLC who progressed on an anti-PD-1/PD-L1 containing therapy. Available from: https://ClinicalTrials.gov/show/NCT03334617. Accessed June 16, 2018.

\section{Publish your work in this journal}

Drug Design, Development and Therapy is an international, peerreviewed open-access journal that spans the spectrum of drug design and development through to clinical applications. Clinical outcomes, patient safety, and programs for the development and effective, safe, and sustained use of medicines are the features of the journal, which has also been accepted for indexing on PubMed Central. The manuscript management system is completely online and includes a very quick and fair peer-review system, which is all easy to use. Visit http://www.dovepress.com/testimonials.php to read real quotes from published authors.

Submit your manuscript here: http://www.dovepress.com/drug-design-development-and-therapy-journal 